\title{
One-year clinical study of NeuroRegen scaffold implantation following scar resection in complete chronic spinal cord injury patients
}

\author{
Zhifeng Xiao ${ }^{1 \dagger}$, Fengwu Tang ${ }^{2 \dagger}$, Jiaguang Tang ${ }^{3 \dagger}$, Huilin Yang $^{4 \dagger}$, Yannan Zhao ${ }^{1}$, Bing Chen ${ }^{1}$, \\ Sufang Han ${ }^{1}$, Nuo Wang ${ }^{1}$, Xing $\mathrm{Li}^{1}$, Shixiang Cheng ${ }^{2}$, Guang $\mathrm{Han}^{2}$, Changyu Zhao ${ }^{2}$, \\ Xiaoxiong Yang ${ }^{3}$, Yumei Chen ${ }^{5}$, Qin $\mathrm{Shi}^{4}$, Shuxun Hou ${ }^{3 *}$, Sai Zhang ${ }^{2 \ddagger}$ \& Jianwu Dai ${ }^{1 \mathrm{II}}$ \\ ${ }^{1}$ State Key Laboratory of Molecular Developmental Biology, Institute of Genetics and Developmental Biology, Chinese Academy of Sciences, \\ Beijing 100190, China; \\ ${ }^{2}$ Neurology and Neurosurgery Hospital, Affiliated Hospital of Logistics University of Chinese People's Armed Police Forces (CAPF), Tianjin \\ 300162, China; \\ ${ }^{3}$ Department of Orthopaedics, First Affiliated Hospital of People's Liberation Army (PLA) General Hospital, Beijing 100048, China; \\ ${ }^{4}$ Department of Orthopaedics, The First Affiliated Hospital of Soochow University, Suzhou 215006, China; \\ ${ }^{5}$ Department of Nerve Electrophysiology, First Affiliated Hospital of People's Liberation Army (PLA) General Hospital, \\ Beijing 100048, China
}

Received May 26, 2016; accepted June 6, 2016; published online June 16, 2016

\begin{abstract}
The objective of this clinical study was to assess the safety and feasibility of the collagen scaffold, NeuroRegen scaffold, one year after scar tissue resection and implantation. Scar tissue is a physical and chemical barrier that prevents neural regeneration. However, identification of scar tissue is still a major challenge. In this study, the nerve electrophysiology method was used to distinguish scar tissue from normal neural tissue, and then different lengths of scars ranging from $0.5-4.5 \mathrm{~cm}$ were surgically resected in five complete chronic spinal cord injury (SCI) patients. The NeuroRegen scaffold along with autologous bone marrow mononuclear cells (BMMCs), which have been proven to promote neural regeneration and SCI recovery in animal models, were transplanted into the gap in the spinal cord following scar tissue resection. No obvious adverse effects related to scar resection or NeuroRegen scaffold transplantation were observed immediately after surgery or at the 12-month follow-up. In addition, patients showed partially autonomic nervous function improvement, and the recovery of somatosensory evoked potentials (SSEP) from the lower limbs was also detected. The results indicate that scar resection and NeuroRegen scaffold transplantation could be a promising clinical approach to treating SCI.
\end{abstract}

NeuroRegen scaffold, chronic spinal cord injury, scar resection, collagen scaffold transplantation, bone marrow mononuclear cells, tissue regeneration

Citation: $\quad$ Xiao, Z., Tang, F., Tang, J., Yang, H., Zhao, Y., Chen, B., Han, S., Wang, N., Li, X., Cheng, S., Han, G., Zhao, C., Yang, X., Chen, Y., Shi, Q., Hou, S., Zhang, S., and Dai, J. (2016). One-year clinical study of NeuroRegen scaffold implantation following scar resection in complete chronic spinal cord injury patients. Sci China Life Sci 59, 647-655. doi: 10.1007/s11427-016-5080-z

\footnotetext{
$\dagger$ Contributed equally as the first authors

*Corresponding author (email: hsxortho@ hotmail.com )

†Corresponding author (email: zhangsai718@vip.126.com)

ICCorresponding author (email: jwdai@genetics.ac.cn)
} 


\section{INTRODUCTION}

Spinal cord injury (SCI) results in the permanent impairment or loss of voluntary motor function and sensation below the level of injury, and effective treatment remains one of the most daunting challenges in neuroscience. The annual incidence of SCI is about 40 cases per million people, mainly due to motor vehicle crashes, falls, sports, and violent acts (Chen et al., 2013). Primarily affecting young adults, SCI tremendously impacts their personal, social, and professional lives, leading to considerable financial, emotional, and psychological burdens on the patients and their families (Selvarajah et al., 2014). The current therapeutic methods for SCI mainly aim to prevent further damage to the injured spinal cord and include operative decompression, injured lesion stabilization and fusion, and rehabilitation (Wilson et al., 2013). Although these approaches extend patient's lifespan, there are no clinical treatments targeting neural regeneration following SCI (Garcia-Altes et al., 2012).

Nerve tissue in the injured mammalian peripheral nervous system exhibits the capacity to lead axons to their synaptic targets based on the removal of myelin debris by immune cells and cytokine secretion by Schwann cells (Schmidt and Leach, 2003). However, SCI leads to scar formation composed of myelin, cellular debris, microglia, astrocytes, oligodendrocytes, meningeal fibroblasts, and extracellular matrix molecules, all of which hinder the regeneration of axons across the injured site (Bahr et al., 1995; Hermanns et al., 2001). Molecules expressed by reactive cells in the scar, such as chondroitin sulfate proteoglycans (CSPGs), contribute to the scar's effects on axon regrowth (McKeon et al., 1995). These molecules exert their inhibitory effects by binding to the axon's cell surface or antagonizing trophic factors and cell adhesion molecules that are essential for axonal regeneration (Levi et al., 1994). Hence, scars are regarded as both a physical and a chemical barrier that prevent nerve regeneration following SCI (Fawcett and Asher, 1999; Yiu and He, 2006).

Many studies have modulated the gliotic scarring response by enzymatic digestion, antibody blocking, or clonal deletion of specific glial scar molecules to promote axon regeneration and functional SCI recovery (Bradbury et al., 2002; Goldshmit et al., 2004; Shearer et al., 2003). Although these biochemical approaches show promise, the therapeutic effects are relatively limited because of the multiple molecule candidates and complicated scar microenvironment. Compared to biochemically altering gliotic responses, surgical scar resection is a more direct approach to eliminate the inhibitory effect of scarring on nerve regeneration after SCI. However, a method for identifying the scar tissue in SCI patients has not been reported, and the resection procedure may lead to further injury if the scar tissue is not precisely identified (Rasouli et al., 2009). In this work, intraoperative neurophysiological monitoring was used to distinguish the scar tissue from normal neural tissue. The combination of intraoperative neurophysiological monitoring with surgical scar resection may provide a safe and effective method to eliminate the inhibitory effects of scarring in patients with chronic SCI.

Biomaterial scaffolds can be used to bridge the lesion site in patients with SCI, providing a structural platform to facilitate axonal growth and also a vehicle to deliver stem cells and functional biomolecules to favorably reconstruct the microenvironment at the injury site (Haggerty and Oudega, 2013). A linearly ordered collagen scaffold termed NeuroRegen scaffold was found to induce axonal growth along collagen fibers and inhibit scar formation after implantation in animal studies. In the past 10 years, the NeuroRegen scaffold has been used to deliver multiple functional molecules such as neurotropic factors and antibodies to the injured site in SCI animal models. Newborn neurons, electrical conductivity, re-myelination, and motor function recovery were observed in both rat and canine SCI models (Han et al., 2009; Han et al., 2010; Han et al., 2015; Li et al., 2015; Lin et al., 2006). The NeuroRegen scaffold product standard has been established and the third party inspection of safety evaluation by the National Institute of Food and Drug Control has been completed in order to lay the foundation for the clinical study.

Bone marrow is regarded as a source of multiple stem and progenitor cells, and bone marrow-derived mesenchymal cells can promote SCI recovery by producing numerous neurotropic factors or immune cytokines (Majka et al., 2001). Since 2005, a number of clinical trials have evaluated the safety and efficacy of autologous bone marrow cell transplantation in acute and chronic SCI patients (Deda et al., 2008; Geffner et al., 2008; Jarocha et al., 2014; Karamouzian et al., 2012; Park et al., 2005; Sykova et al., 2006; Yoon et al., 2007).

In this study, we first took advantage of intraoperative neurophysiological monitoring to identify and surgically resect scar tissues ranging from $0.5-4.5 \mathrm{~cm}$ in five complete chronic SCI patients, and then the NeuroRegen scaffold with autologous bone marrow mononuclear cells (BMMCs) was implanted into the resection sites. The clinical study was approved by the ethics committee and registered on the National Institute of Health database (ClinicalTrials.gov: NCT02352077). All procedures performed in this study were in accordance with the ethical standards of a human clinical study. We analyzed the safety and potential efficacy of scar resection and scaffold transplantation during a one-year period. The results provide the first evidence that scar resection with nerve electrophysiology monitoring and subsequent biomaterial scaffold implantation might be a promising clinical approach to rebuilding a regenerative microenvironment for SCI repair. 


\section{RESULTS}

\section{Linear and ordered structure of NeuroRegen scaffold fibers}

The NeuroRegen scaffold fibers fabricated in this study were arranged linearly to effectively guide the orientated growth of axons (Figure 1). There are many tiny collagen fibers in a single fiber, which may increase the space for cell attachment and ingrowth. The biological safety of the NeuroRegen scaffold was evaluated by the National Institute of Food and Drug Control, which meets the Chinese Criterion of Medical Device GB16886.

\section{Patients}

Five patients with chronic traumatic SCI who met the inclusion criteria were enrolled in our study. Their demographic information is shown in Table 1. All patients were classified as American Spinal Injury Association (ASIA) Impairment Scale grade A and had complete injuries in cervical or thoracic spinal cord segments.

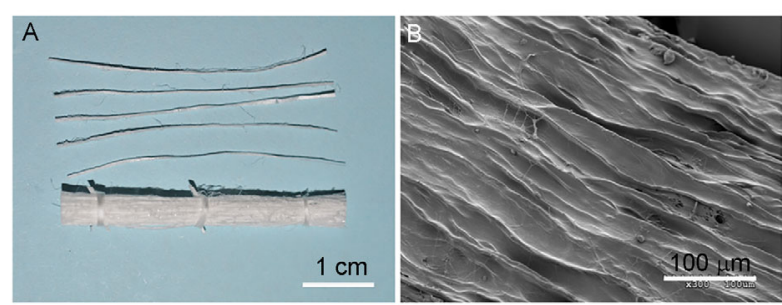

Figure 1 Linear and ordered structure of the NeuroRegen scaffold. A, Photo of NeuroRegen scaffold. Scale bar $=1 \mathrm{~cm}$. B, SEM image exhibiting microstructure of a single NeuroRegen scaffold fiber. Scale bar=100 $\mu \mathrm{m}$.

\section{Scar resection under neural electrophysiology monitoring}

After opening the dura mater, the injured spinal cords were filled with scar tissue. Because it is difficult to distinguish the boundaries between normal spinal cord tissue and scar tissue, we developed a new strategy using somatosensory evoked potentials (SSEP) and motor evoked potentials (MEP) to identify rostral and caudal edges of the scar, and then the scar was resected. The lengths of scars in individual SCI patient ranged from $0.5-4.5 \mathrm{~cm}$ (Table 2). Following scar tissue resection, the NeuroRegen scaffold with BMMCs was transplanted into the spinal cord gap (Figure 2). Histological examination of the resected scar tissue showed strong positive immunohistochemical staining for CSPGs and vimentin, and negative immunohistochemical staining for NF, nestin, Tuj-1, and S100 (Figure 3). These results demonstrated that the dissected tissue mainly consisted of scar tissue.

\section{Safety assessment of scar resection and NeuroRegen scaffold transplantation}

Safety is a major concern regarding scar resection and NeuroRegen scaffold transplantation. Despite the different lengths of dissected scars, there were no increases in the ASIA Impairment Scale grade, sensation, or motor level after surgery (Table 2), and no obvious neurological deterioration was detected during follow-up. Adverse effects including fever, infection, allergic reaction, and perioperative complications (anesthesia-related complications) were observed to assess the safety. There were no significant adverse effects related to scar resection or NeuroRegen scaffold transplantation immediately after the surgery or 12
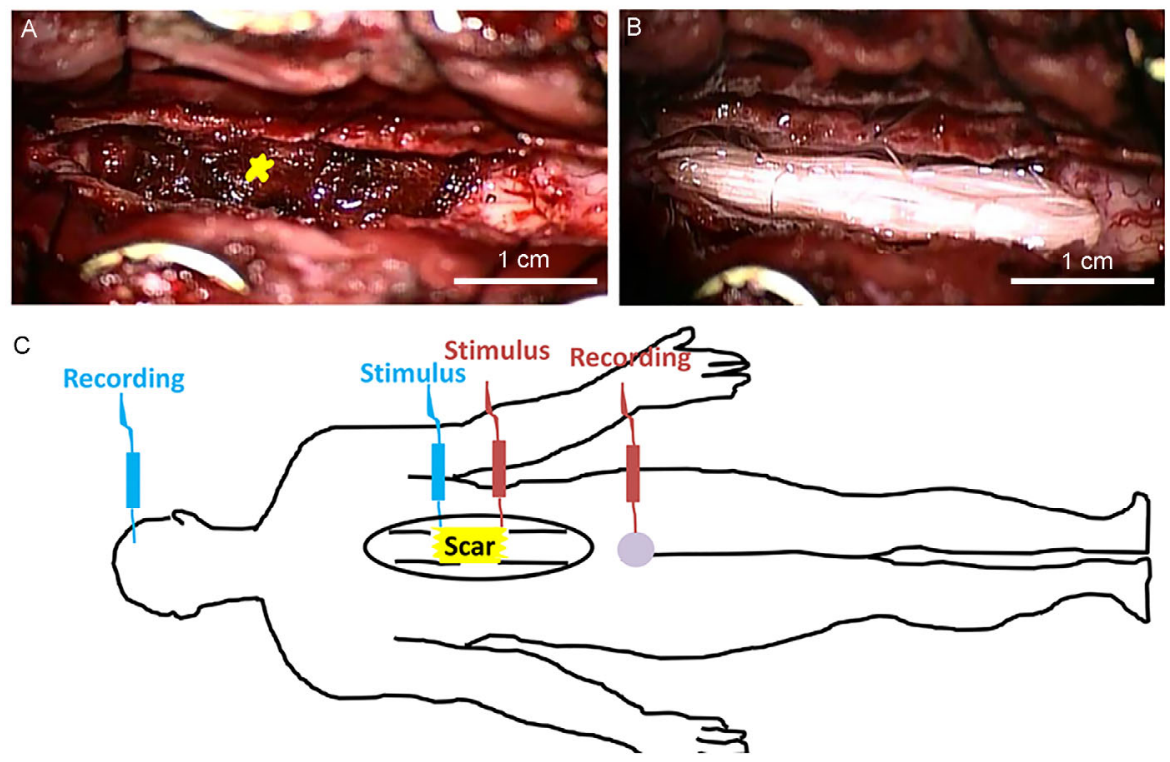

Figure 2 Surgical procedures and scar resection diagram. A, The spinal cord was exposed after opening the dura mater, and the asterisk (*) indicates the gap after scar tissue resection. Scale bar $=1 \mathrm{~cm}$. B, The NeuroRegen scaffold with BMMCs was transplanted into the spinal cord gap. Scale bar=1 cm. C, Diagram of the scar resection guided by SSEP (blue color) and MEP (red color). 

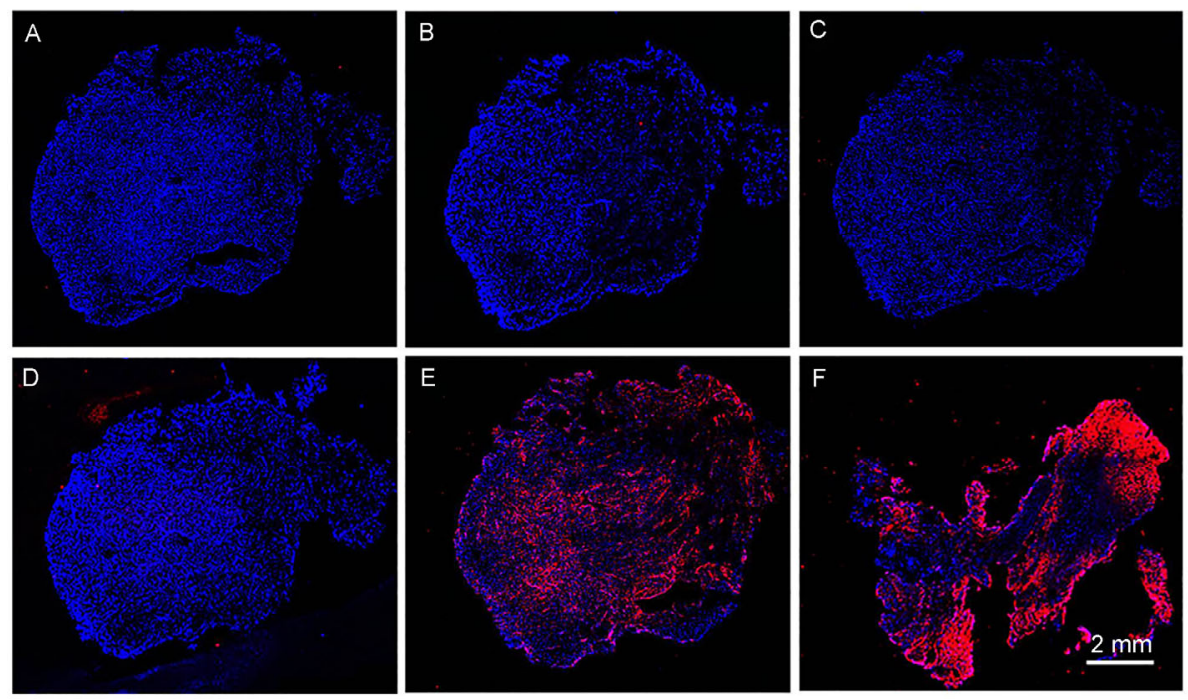

Figure 3 Immunofluorescence staining of dissected scar tissue. The antibodies in panels A-F are neurofilament $200 \mathrm{kD}$ (NF), nestin, $\beta$-tubulin III (Tuj-1), S100 protein, chondroitin sulfate proteoglycans (CSPGs), and vimentin, respectively. Scale bar=2 $\mathrm{mm}$.

months postoperatively (Table 3). These results demonstrate the safety of scar resection and NeuroRegen scaffold transplantation.

\section{SCI patient recovery}

Autonomic neural function was assessed after surgery. Sexual arousal was achieved in two cases, showing improved penis reflex erection 2 month after surgery. Diminished sweating in skin below the injury level was partially recovered in three cases. More importantly, when low limb SSEP was performed, two cases began to show SSEP recovery at 6 months postoperatively with normal latency and lower amplitude, and this persisted until the end of the observation period (Table 4).

\section{DISCUSSION}

Five complete injury patients (ASIA A) were enrolled in this clinical study. Neural electrophysiological studies showed that there were no MEP detected in muscles below the injury level and no SSEP conducted from the ankle to the cortex through the injury level. Magnetic resonance imaging (MRI) showed complete injury in all five patients, confirming the total loss of motor or sensory functions below the injury site. There is little evidence to support functional recovery after complete SCI. Conventional treatments such as drug administration or rehabilitation exercises have no significant effects on neurological recovery in chronic SCI.

SCI is mainly caused by trauma such as car accidents and falls, often initiating a cascade of biochemical reactions. Following the initial insult, a variety of inflammatory and cytotoxic mediators is released at the injured site, resulting in secondary damage to the spinal cord. This leads to con-
Table 1 Patient demographic and clinical features ${ }^{\text {a) }}$

\begin{tabular}{cccccc}
\hline Patient & Sex & Age & Months post SCI & SCI level & ASIA grade \\
\hline 1 & Male & 27 & 2 & T11-T12 & A \\
2 & Male & 53 & 14 & C6-C7 & A \\
3 & Male & 51 & 7 & T9-T10 & A \\
4 & Female & 56 & 32 & T10-T12 & A \\
5 & Male & 29 & 10 & T8-T9 & A \\
\hline
\end{tabular}

a) ASIA, American Spinal Injury Association; SCI, spinal cord injury.

Table 2 Pre- and postoperative neural function assessment ${ }^{\text {a) }}$

\begin{tabular}{ccccccc}
\hline \multirow{2}{*}{ Patient } & Glial scar length & \multicolumn{2}{c}{ ASIA grade } & & \multicolumn{2}{c}{ Sensation level } \\
\cline { 3 - 4 } \cline { 7 - 8 }$(\mathrm{cm})$ & 2.8 & Before & After & & Before & After \\
\hline 1 & 2.8 & $\mathrm{~A}$ & $\mathrm{~A}$ & & T11 & T11 \\
2 & 0.5 & $\mathrm{~A}$ & $\mathrm{~A}$ & & $\mathrm{~T} 3$ & $\mathrm{~T} 3$ \\
3 & 3.5 & $\mathrm{~A}$ & $\mathrm{~A}$ & & $\mathrm{~T} 9$ & $\mathrm{~T} 9$ \\
4 & 3.0 & $\mathrm{~A}$ & $\mathrm{~A}$ & & $\mathrm{~T} 10$ & $\mathrm{~T} 10$ \\
5 & 4.5 & $\mathrm{~A}$ & $\mathrm{~A}$ & & $\mathrm{~T} 8$ & $\mathrm{~T} 8$ \\
\hline
\end{tabular}

a) ASIA, American Spinal Injury Association.

Table 3 Adverse effects assessment after surgery

\begin{tabular}{|c|c|c|c|c|c|}
\hline Complications & Patient 1 & Patient 2 & Patient 3 & Patient 4 & Patient 5 \\
\hline Infection & - & - & - & - & - \\
\hline $\begin{array}{l}\text { Increased level of C-reactive } \\
\text { protein }\end{array}$ & - & - & - & - & - \\
\hline Increased leukocytosis & - & - & - & - & - \\
\hline Allergic reaction or shock & - & - & - & - & - \\
\hline Perioperative complications & - & - & - & - & - \\
\hline
\end{tabular}

Table 4 Pre- and postoperative SSEP assessment ${ }^{\text {a) }}$

\begin{tabular}{ccccccc}
\hline \multirow{2}{*}{ Patient } & & \multicolumn{2}{c}{ Before surgery } & & \multicolumn{2}{c}{6 months after surgery } \\
\cline { 3 - 4 } \cline { 6 - 7 } & & Latency & Amplitude & & Latency & Amplitude \\
\hline 4 & $\begin{array}{c}\text { Left tibial } \\
\text { malleolus }\end{array}$ & 0 & 0 & & $45.0 \mathrm{~ms}$ & $0.94 \mu \mathrm{v}$ \\
5 & $\begin{array}{c}\text { Right tibial } \\
\text { malleolus }\end{array}$ & 0 & 0 & & $42.8 \mathrm{~ms}$ & $0.43 \mu \mathrm{v}$ \\
\hline
\end{tabular}

a) SSEP, somatosensory evoked potential. 
tinued and pervasive cell death and tissue damage. Furthermore, astrocytes become hypertrophic in response to SCI and produce CSPGs that form a dense scar at the injury site. Many reports have demonstrated that glial scars are impediments to neural regeneration, making them primary targets for therapeutic strategies after SCI (Cregg et al., 2014; Lukovic et al., 2015; Silver and Miller, 2004). Chondroitinase $\mathrm{ABC}(\mathrm{ChABC})$ can enzymatically digest the glycosaminoglycan side-chains of CSPGs, and ChABC treatment could improve glial scar digestion to reduce the lesion size and increase neural regeneration, which might further promote functional recovery in rat SCI (Barritt et al., 2006; Bradbury and Carter, 2011; Zhao and Fawcett, 2013). However, studies assessing the efficacy of ChABC treatment have only been performed in small animal models. Furthermore, ChABC has poor thermostability and is synthesized from Escherichia coli; it may lose its enzymatic activity at body temperature and could carry immunological risk. Therefore, translating $\mathrm{ChABC}$ treatment into a clinical SCI therapy remains a challenge (Bradbury and Carter, 2011; Tester et al., 2007).

Surgical scar removal is a more direct approach to eliminating the inhibitory effect of scar tissue. Tabakow et al. removed yellowish scar tissue under an operating microscope, but the glial scar was tightly connected with normal neural tissue, and it was difficult to accurately distinguish the scar from normal neural tissue under the microscope (Tabakow et al., 2014). MRI or functional MRI is considered the best method for determining the extent of spinal cord injury (Vedantam et al., 2014), but it has been reported that the MRI estimates of lesion length are not always consistent with histological assessment in rat SCI (Ditor et al., 2008). In our study, SSEP and MEP were used to determine the rostral and caudal edges of the scar tissue, respectively. By the neural electrophysiology method, the length of the scar was ascertained and removed. We found that the lesion length estimated by MRI was often smaller than intraoperative neurophysiological monitoring results. No neurological deterioration or spine instability was detected after scar resection. The immunostaining results showed that the dissected tissue mainly consisted of scar, and no nerve fibers or neural cells were detected in the tissue, which indicated that the scar was removed safely without further damaging neural function. Resecting the glial scar removes a physical and chemical barrier to neural regeneration and may promote neural repair. Thus, the length of scar was determined in vivo and it was safely removed under neural electrophysiology monitoring in complete SCI patients. For incomplete SCI patients, spontaneous plasticity events in spared neuronal circuits may occur post-traumatically, including intact or injured axon collateral sprouting or synaptic rearrangement (Onifer et al., 2011; Perez, 2015; Tansey, 2010), which may result in scar tissue mixed together with normal neural tissue. For this reason, dissecting scar tissue in incomplete SCI patients is still a challenge.
Collagen scaffolds are considered a suitable biomaterial for guiding neural regeneration because of their low antigenicity and excellent biocompatibility and biodegradability. Different types of collagen scaffolds have been used to repair SCI in animal models, including collagen tubes, fibers, membranes, and gels. A growing body of work shows that collagen scaffolds are suitable for guiding neural regeneration (Sindou, 2001; Stang et al., 2005; Yoshii et al., 2004). In our previous study, the NeuroRegen scaffold was prepared from bovine aponeurosis, which mainly consists of linearly ordered collagen fibers. When the scaffold was transplanted into complete SCI rat and canine models, it successfully guided neurite outgrowth along its fibers and decreased scar formation (Fan et al., 2010; Han et al., 2009; Han et al., 2010; Han et al., 2015; Li et al., 2013; Lin et al., 2006). Based on these preclinical results, the clinical study was approved by the ethics committee and registered on the National Institute of Health database. All procedures performed in this study were in accordance with the ethical standards of human clinical study. No obvious adverse symptoms related to the NeuroRegen scaffold transplantation were observed, such as infection, allergic reaction, or perioperative complications. The cerebrospinal fluid did not show any evidence of bacterial or fungal infection. In one case, a slight non-inflammatory fever was observed after surgery, but it was transient and did not exceed $38^{\circ} \mathrm{C}$. After symptomatic treatment, the patient recovered within $72 \mathrm{~h}$. These data show that NeuroRegen scaffold transplantation is safe. Our recent work demonstrated that NeuroRegen scaffold with mesenchymal stem cells could inhibit scar formation, induce newborn neuron production, and promote functional recovery after scar resection in complete transected chronic SCI rats and canines (unpublished data). Following scar tissue resection under electrophysiological monitoring in patients, the BMMCs and NeuroRegen scaffold were transplanted into the spinal cord gap, where they could inhibit new scar formation and provide a suitable microenvironment to guide neural regeneration.

Besides the total loss of motor and sensory function below the injury site, autonomic dysfunction including abnormal blood pressure, heart rate control, sweating, and temperature dysregulation are common clinical consequences of SCI. In our study, sexual arousal and diminished sweating were achieved in patients, indicating that autonomic nervous system function was partially restored. More importantly, recovery of SSEP in tibial malleolus was detected in two cases 6 months after surgery. These results showed that a certain degree of neural regeneration occurred following scar resection and NeuroRegen scaffold transplantation. A longer observation time is necessary to assess the recovery of complete SCI after these procedures.

We found that the lengths of the scars were diverse. The lengths of scars in the five SCI patients ranged from 0.5-4.5 $\mathrm{cm}$. Scar length was not directly correlated with the location of the injured segments or time post-SCI, but might be cor- 
related with SCI patient constitution and injury severity. It has long been recognized that axonal regeneration is the main way to restore function after SCI. However, the rate of axonal elongation may be slower in the central nervous system even under "regeneration enabled" circumstances than in the peripheral nervous system, in which the growth velocity of regenerating peripheral nerves through the scar is $0.25 \mathrm{~mm}$ per day. Furthermore, there is little evidence supporting corticospinal tract (CST) growth into grafts or transplants (Tuszynski and Steward, 2012). It might be difficult to restore function by axonal regeneration after severe SCI in which the length of the scar is often more than one segment of spinal cord $(2 \mathrm{~cm})$. Our recent work showed that NeuroRegen scaffold with active factors increased neuronal differentiation of the transplanted or endogenous neural stem cells to produce different types of neurons throughout the lesion area. These newly generated relay neurons may further rebuild the synaptic connections with each other or with the host spinal neurons to improve locomotion outcomes in SCI animals (Li et al., 2015; Li et al., 2013). Thus, rebuilding neuronal relays at the injury site would be a more efficient way to repair SCI compared with inducing axonal regrowth (Xiao et al., 2016).

Following SCI, an inhibitory environment for neural regeneration develops at the injury site. Many reports have demonstrated that scaffolds can rebuild the microenvironment at the injured site. The NeuroRegen scaffold bridges the gap of the lesion and acts as a vehicle to deliver stem cells or biomolecules to promote neural regeneration. In our previous study, a functional biomaterial consisting of NeuroRegen scaffold, biologically active molecules (neurotrophic factors or antagonists to myelin-associated inhibitors), and stem cells was developed that greatly promoted neural regeneration and functional recovery in SCI animals (Han et al., 2010; Han et al., 2015; Li et al., 2015; Li et al., 2013). In future work, biologically active molecules or stem cells could be added to the NeuroRegen scaffold for clinical SCI repair.

\section{MATERIALS AND METHODS}

\section{Patient selection}

Five patients with complete SCI (ASIA Impairment Scale grade A) were included in this study in three hospitals: Affiliated Hospital of Logistics University of CAPF, The First Affiliated Hospital of Soochow University, and First Affiliated Hospital of PLA General Hospital. The main inclusion and exclusion criteria of the study were as follows:

\section{Inclusion Criteria}

(i) Male or female, 18-65 years old. (ii) Complete spinal cord injury at the cervical or thoracic level (C5-T12). (iii) ASIA A classification with no significant further improve- ment. (iv) Patients signed informed consent. (v) Ability and willingness to regularly visit the hospital and follow-up during the study.

\section{Exclusion Criteria}

(i) Serious complications. (ii) History of life-threatening allergic or immune-mediated reaction. (iii) Clinically significant abnormalities in routine laboratory examinations. (iv) Lactating and pregnant woman. (v) Participated in any other clinical trials within 3 months before enrollment. (vi) Poor compliance, difficultly completing the study requirements.

\section{Preparation and biological safety evaluation of the NeuroRegen scaffold}

NeuroRegen scaffolds were prepared from bovine aponeurosis using previously described methods, with modifications (Lin et al., 2006). Briefly, fresh bovine aponeurosis were harvested and rinsed with cold distilled water, and residual muscles, connective tissue, and fat were carefully removed. The samples were treated with Tri (n-butyl) phosphate (TnBP), hypertonic solution, and enzyme to further remove fat, cellular components, and soluble proteins. The samples were then repeatedly rinsed to completely remove the residual agents and freeze-dried. The scaffolds were critical point dried and sputter coated with gold prior to SEM imaging (S-3000N SEM; Hitachi, Japan).

Acute toxicity, subchronic toxicity, allergen detection, genetic toxicity, intradermal irritation, cytotoxicity, hemolytic toxicity, and degradability of the NeuroRegen scaffold were evaluated by the National Institute of Food and Drug Control according to Chinese Criterion of Medical Device GB16886.

\section{BMMC preparation}

Before the procedure, patients underwent tests for hematology, blood biochemistry, urine microbiology, and screening for human immunodeficiency virus, human T-cell lymphotropic virus, Chagas disease, and hepatitis $\mathrm{B}$ and $\mathrm{C}$. The isolation of BMMCs from bone marrow suspension was then performed. Bone marrow $(50 \mathrm{~mL})$ was harvested under local anesthesia from the posterior iliac crest and was immediately heparinized. The bone marrow was then sedimented using hydroxyethyl starch for erythrocyte reduction. Hydroxyethyl starch was added to the bone marrow until a final concentration of $3 \%$ was reached. After erythrocytes had sedimented, the upper cell suspension was collected into $50-\mathrm{mL}$ tubes and centrifuged $700 \mathrm{~g}$ at $25^{\circ} \mathrm{C}$ for $10 \mathrm{~min}$. The supernatant was discarded, and the cell suspension was washed with physiological salt solution. The BMMCs were diluted to a final volume of $0.5 \mathrm{~mL}$ and used for transplantation. The cell number for transplantation was about $1 \times 10^{9}$ cells. 


\section{Surgical and BMMC transplantation procedures}

After identification of the injury level with MRI, the patient was placed under general anesthesia in a prone position. A midline skin incision was made, followed by paravertebral muscle dissection and laminectomy. Under an operating microscope, an adhesion outside the injured dura was removed. A midline durotomy was performed, followed by sharp dissection of the post-traumatic adhesions between the spinal cord surface and dura. The injury area mainly consisted of scar tissue and a cavity. The scar tissue was resected under nerve electrophysiology monitoring, and specimens were taken for histology. Next, BMMCs were added to the NeuroRegen scaffold and grafted into the transected spinal cord gap to bridge the defect. The dura was closed with absorbable sutures.

\section{Scar tissue resection under neural electrophysiology monitoring}

The texture of the scar tissue is different from the normal spinal cord structure, and the middle of the scar may have necrotic tissue or cavities. The boundaries of the normal spinal cord and scar tissue are generally determined according to the neural electrophysiology (Figure 2C). SSEP and MEP were used to determine the rostral and caudal ends of the scar tissue, respectively. The stimulation electrodes of electromyography (XLTEK, Canada) were placed near the rostral side of the spinal cord injury site, and the recording electrodes were placed on the scalp before moving the stimulation electrodes. If there was an SSEP response, the position of the stimulation electrodes was on normal spinal cord tissue and if there was no SSEP response, the position was scar tissue. For caudal scar tissue, the stimulation electrodes were placed near the caudal end of the SCI, and the recording electrodes were placed into the sphincter ani externus before moving the stimulation electrodes. If there was an MEP response, the position of the stimulation electrodes was normal spinal cord tissue, and if there was no MEP response, the position was scar tissue. After detecting the edge of the rostral and caudal ends of the scar tissue, the scar was dissected with an electronic knife and removed under the operating microscope.

\section{Immunocytochemistry}

Tissue samples taken from scar tissue were formalin-fixed overnight at $4^{\circ} \mathrm{C}$ and transferred to $20 \%$ sucrose (overnight at $4^{\circ} \mathrm{C}$ ) and then $30 \%$ sucrose $\left(72 \mathrm{~h}\right.$ at $\left.4{ }^{\circ} \mathrm{C}\right)$. The samples were then embedded in Tissue-Tek O.C.T. compound (Sakura Finetechnical Co., Japan) and sectioned on a cryostat set at $10-\mu \mathrm{m}$ thickness (Leica Microsystems $\mathrm{GmbH}$, Germany). The slides were fixed in acetone for $15 \mathrm{~min}$ at $4^{\circ} \mathrm{C}$ and then incubated in phosphate-buffered saline (PBS) containing $5 \%$ bovine serum albumin (BSA) with $0.1 \%$ Triton $\mathrm{X}-100$ for $1 \mathrm{~h}$ at $25^{\circ} \mathrm{C}$ room temperature. The samples were incubated with primary antibodies overnight at $4{ }^{\circ} \mathrm{C}$ : mouse monoclonal antibody against neurofilament $200 \mathrm{kDa}(\mathrm{NF}$, 1:200, ab3966, Abcam, UK), S100 protein (1:100, ZM-0224, ZSGB-Bio, China), ßIII tubulin (Tuj-1, 1:500, 05-559, Millipore, USA), nestin (1:500, MAB353, Millipore), CSPG (1:500, ab11570, Abcam), and vimentin (1:500, V6630, Sigma, USA). They were then incubated with Alexa Fluor 568-conjugated donkey anti-mouse secondary antibody (A10037, 1:800, Invitrogen, USA) for $1 \mathrm{~h}$ at RT. Cell nuclei were stained with Hoechst 33342 (1:1000, B2261, Sigma), and all images were taken with a Leica TCS SP8 confocal microscope.

\section{Patient follow-up}

Patients underwent rehabilitation for 6 months after surgery. Regular clinical and neurological assessments were performed for at least 12 months. At each follow-up, complete clinical and neurological evaluations and ASIA Impairment Scale assessment were conducted before and 1, 3, 6, and 12 months after surgery. SSEP testing was also carried out with tibial nerve stimulation for evaluation of the lower limbs, registration in the ankle, popliteal fossa, and scalp $\left(\mathrm{Cz}^{\prime}\right.$ to Fpz) regions; the scalp electrodes were placed in the parietal and frontal regions for upper limb SSEP and at the vertex for lower limb SSEP. A 100-ms square wave electrical pulse was delivered at intensities strong enough to cause a thumb twitch. We identified a $\mathrm{P} 40$ potential in response to target stimuli that could affect early cortical SSEP.

\section{Compliance and ethics}

The author(s) declare that they have no conflict of interest. The clinical study was approved by the ethics committee of each hospital and registered on the National Institute of Health database (ClinicalTrials.gov: NCT02352077). All procedures performed in studies involving human participants were in accordance with the ethical standards of each hospital and with the 1964 Helsinki declaration and its later amendments or comparable ethical standards. Informed consent was obtained from each patient to participate in the study and publish the results.

Compliance and ethics The author(s) declare that they have no conflict of interest.

Acknowledgements This work was supported by grants from the "Stem Cell and Regenerative Medicine Strategic Priority Research Program of the Chinese Academy of Sciences" (Grant No. XDA01030000) and the Key Research Program of the Chinese Academy of Sciences (Grant No. ZDRW-ZS-2016-2).

Bahr, M., Przyrembel, C., and Bastmeyer, M. (1995). Astrocytes from adult rat optic nerves are nonpermissive for regenerating retinal ganglion cell axons. Exp Neurol 131, 211-220.

Barritt, A.W., Davies, M., Marchand, F., Hartley, R., Grist, J., Yip, P., McMahon, S.B., and Bradbury, E.J. (2006). Chondroitinase ABC 
promotes sprouting of intact and injured spinal systems after spinal cord injury. J Neurosci 26, 10856-10867.

Bradbury, E.J., and Carter, L.M. (2011). Manipulating the glial scar: Chondroitinase $\mathrm{ABC}$ as a therapy for spinal cord injury. Brain Res Bull 84, 306-316.

Bradbury, E.J., Moon, L.D., Popat, R.J., King, V.R., Bennett, G.S., Patel, P.N., Fawcett, J.W., and McMahon, S.B. (2002). Chondroitinase ABC promotes functional recovery after spinal cord injury. Nature 416, 636-640.

Chen, Y., Tang, Y., Vogel, L.C., and Devivo, M.J. (2013). Causes of spinal cord injury. Top Spinal Cord Inj Rehabil 19, 1-8.

Cregg, J.M., DePaul, M.A., Filous, A.R., Lang, B.T., Tran, A., and Silver, J. (2014). Functional regeneration beyond the glial scar. Exp Neurol 253, 197-207.

Deda, H., Inci, M.C., Kurekci, A.E., Kayihan, K., Ozgun, E., Ustunsoy, G.E., and Kocabay, S. (2008). Treatment of chronic spinal cord injured patients with autologous bone marrow-derived hematopoietic stem cell transplantation: 1-year follow-up. Cytotherapy 10, 565-574.

Ditor, D.S., John, S., Cakiroglu, J., Kittmer, C., Foster, P.J., and Weaver, L.C. (2008). Magnetic resonance imaging versus histological assessment for estimation of lesion volume after experimental spinal cord injury. Laboratory investigation. J Neurosurg Spine 9, 301-306.

Fan, J., Xiao, Z.F., Zhang, H.T., Chen, B., Tang, G.Q., Hou, X.L., Ding, W.Y., Wang, B., Zhang, P., Dai, J.W., and Xu, R.X. (2010). Linear ordered collagen scaffolds loaded with collagen-binding neurotrophin-3 promote axonal regeneration and partial functional recovery after complete spinal cord transection. J Neurotrauma 27, 1671-1683.

Fawcett, J.W., and Asher, R.A. (1999). The glial scar and central nervous system repair. Brain Res Bull 49, 377-391.

Garcia-Altes, A., Perez, K., Novoa, A., Suelves, J.M., Bernabeu, M., Vidal, J., Arrufat, V., Santamarina-Rubio, E., Ferrando, J., Cogollos, M., Cantera, C.M., and Luque, J.C. (2012). Spinal cord injury and traumatic brain injury: a cost-of-illness study. Neuroepidemiology 39, 103-108.

Geffner, L.F., Santacruz, P., Izurieta, M., Flor, L., Maldonado, B., Auad, A.H., Montenegro, X., Gonzalez, R., and Silva, F. (2008). Administration of autologous bone marrow stem cells into spinal cord injury patients via multiple routes is safe and improves their quality of life: comprehensive case studies. Cell Transplant 17, 1277-1293.

Goldshmit, Y., Galea, M.P., Wise, G., Bartlett, P.F., and Turnley, A.M. (2004). Axonal regeneration and lack of astrocytic gliosis in EphA4-deficient mice. J Neurosci 24, 10064-10073.

Haggerty, A.E., and Oudega, M. (2013). Biomaterials for spinal cord repair. Neurosci Bull 29, 445-459.

Han, Q.Q., Sun, W.J., Lin, H., Zhao, W.X., Gao, Y., Zhao, Y.N., Chen, B., Xiao, Z.F., Hu, W., Li, Y., Yang, B., and Dai, J.W. (2009). Linear ordered collagen scaffolds loaded with collagen-binding brain-derived neurotrophic factor improve the recovery of spinal cord injury in rats. Tissue Eng Part A 15, 2927-2935.

Han, Q.Q., Jin, W., Xiao, Z.F., Ni, H.B., Wang, J.H., Kong, J., Wu, J., Liang, W.B., Chen, L., Zhao, Y.N., Chen, B., and Dai, J.W. (2010). The promotion of neural regeneration in an extreme rat spinal cord injury model using a collagen scaffold containing a collagen binding neuroprotective protein and an EGFR neutralizing antibody. Biomaterials 31, 9212-9220.

Han, S.F., Wang, B., Jin, W., Xiao, Z.F., Li, X., Ding, W.Y., Kapur, M., Chen, B., Yuan, B.Y., Zhu, T.S., Wang, H.D., Wang, J., Dong, Q., Liang, W.B., and Dai, J.W. (2015). The linear-ordered collagen scaffold-BDNF complex significantly promotes functional recovery after completely transected spinal cord injury in canine. Biomaterials 41, 89-96.

Hermanns, S., Klapka, N., and Muller, H.W. (2001). The collagenous lesion scar--an obstacle for axonal regeneration in brain and spinal cord injury. Restor Neurol Neurosci 19, 139-148.

Jarocha, D., Milczarek, O., Kawecki, Z., Wendrychowicz, A., Kwiatkowski, S., and Majka, M. (2014). Preliminary study of autologous bone marrow nucleated cells transplantation in children with spinal cord injury. Stem Cells Transl Med 3, 395-404.

Karamouzian, S., Nematollahi-Mahani, S.N., Nakhaee, N., and Eskandary, H. (2012). Clinical safety and primary efficacy of bone marrow mesenchymal cell transplantation in subacute spinal cord injured patients. Clin Neurol Neurosurg 114, 935-939.

Levi, A.D., Guenard, V., Aebischer, P., and Bunge, R.P. (1994). The functional characteristics of Schwann cells cultured from human peripheral nerve after transplantation into a gap within the rat sciatic nerve. J Neurosci 14, 1309-1319.

Li, X., Han, J., Zhao, Y.N., Ding, W.Y., Wei, J.S., Han, S.F., Shang, X.P., Wang, B., Chen, B., Xiao, Z.F., and Dai, J.W. (2015). Functionalized Collagen Scaffold Neutralizing the Myelin-Inhibitory Molecules Promoted Neurites Outgrowth in Vitro and Facilitated Spinal Cord Regeneration in Vivo. Acs Appl Mater Inter 7, 13960-13971.

Li, X.R., Xiao, Z.F., Han, J., Chen, L., Xiao, H.S., Ma, F.K., Hou, X.L., Li, X., Sun, J., Ding, W.Y., Zhao, Y.N., Chen, B., and Dai, J.W. (2013). Promotion of neuronal differentiation of neural progenitor cells by using EGFR antibody functionalized collagen scaffolds for spinal cord injury repair. Biomaterials 34, 5107-5116.

Lin, H., Chen, B., Wang, B., Zhao, Y.N., Sun, W.J., and Dai, J.W. (2006). Novel nerve guidance material prepared from bovine aponeurosis. J Biomed Mater Res A 79, 591-598.

Lukovic, D., Stojkovic, M., Moreno-Manzano, V., Jendelova, P., Sykova, E., Bhattacharya, S.S., and Erceg, S. (2015). Concise Review: Reactive Astrocytes and Stem Cells in Spinal Cord Injury: Good Guys or Bad Guys? Stem Cells 33, 1036-1041.

Majka, M., Janowska-Wieczorek, A., Ratajczak, J., Ehrenman, K., Pietrzkowski, Z., Kowalska, M.A., Gewirtz, A.M., Emerson, S.G., and Ratajczak, M.Z. (2001). Numerous growth factors, cytokines, and chemokines are secreted by human CD34(+) cells, myeloblasts, erythroblasts, and megakaryoblasts and regulate normal hematopoiesis in an autocrine/paracrine manner. Blood 97, 3075-3085.

McKeon, R.J., Hoke, A., and Silver, J. (1995). Injury-induced proteoglycans inhibit the potential for laminin-mediated axon growth on astrocytic scars. Exp Neurol 136, 32-43.

Onifer, S.M., Smith, G.M., and Fouad, K. (2011). Plasticity After Spinal Cord Injury: Relevance to Recovery and Approaches to Facilitate It. Neurotherapeutics 8, 283-293.

Park, H.C., Shim, Y.S., Ha, Y., Yoon, S.H., Park, S.R., Choi, B.H., and Park, H.S. (2005). Treatment of complete spinal cord injury patients by autologous bone marrow cell transplantation and administration of granulocyte-macrophage colony stimulating factor. Tissue Eng 11, 913-922.

Perez, M. (2015). Plasticity in the Corticospinal System after Spinal Cord Injury. J Neurotraum 32, A130-A131.

Rasouli, A., Bhatia, N., Dinh, P., Cahill, K., Suryadevara, S., and Gupta, R. (2009). Resection of glial scar following spinal cord injury. J Orthop Res 27, 931-936.

Schmidt, C.E., and Leach, J.B. (2003). Neural tissue engineering: strategies for repair and regeneration. Annu Rev Biomed Eng 5, 293-347.

Selvarajah, S., Hammond, E.R., Haider, A.H., Abularrage, C.J., Becker, D., Dhiman, N., Hyder, O., Gupta, D., Black, J.H., 3rd, and Schneider, E.B. (2014). The burden of acute traumatic spinal cord injury among adults in the united states: an update. J Neurotrauma 31, 228-238.

Shearer, M.C., Niclou, S.P., Brown, D., Asher, R.A., Holtmaat, A.J., Levine, J.M., Verhaagen, J., and Fawcett, J.W. (2003). The astrocyte/meningeal cell interface is a barrier to neurite outgrowth which can be overcome by manipulation of inhibitory molecules or axonal signalling pathways. Mol Cell Neurosci 24, 913-925.

Silver, J., and Miller, J.H. (2004). Regeneration beyond the glial scar. Nat Rev Neurosci 5, 146-156.

Sindou, M. (2001). Regrowth of the rostral spinal axons into the caudal ventral roots through a collagen tube implanted into hemisected adult rat spinal cord-Comment. Neurosurgery 49, 150-151.

Stang, F., Fansa, H., Wolf, G., and Keilhoff, G. (2005). Collagen nerve conduits-assessment of biocompatibility and axonal regeneration. Bio-Med Mater Eng 15, 3-12.

Sykova, E., Homola, A., Mazanec, R., Lachmann, H., Konradova, S.L., Kobylka, P., Padr, R., Neuwirth, J., Komrska, V., Vavra, V., Stulik, J., and Bojar, M. (2006). Autologous bone marrow transplantation in patients with subacute and chronic spinal cord injury. Cell Transplant $15,675-687$. 
Tabakow, P., Raisman, G., Fortuna, W., Czyz, M., Huber, J., Li, D.Q., Szewczyk, P., Okurowski, S., Miedzybrodzki, R., Czapiga, B., Salomon, B., Halon, A., Li, Y., Lipiec, J., Kulczyk, A., and Jarmundowicz, W. (2014). Functional regeneration of supraspinal connections in a patient with transected spinal cord following transplantation of bulbar olfactory ensheathing cells with peripheral nerve bridging. Cell Transplant 23, 1631-1655.

Tansey, K.E. (2010). Neural plasticity and locomotor recovery after spinal cord injury. Pm\&R 2, S220-S226.

Tester, N.J., Plaas, A.H., and Howland, D.R. (2007). Effect of body temperature on chondroitinase $\mathrm{ABC}$ 's ability to cleave chondroitin sulfate glycosaminoglycans. J Neurosci Res 85, 1110-1118.

Tuszynski, M.H., and Steward, O. (2012). Concepts and methods for the study of axonal regeneration in the CNS. Neuron 74, 777-791.

Vedantam, A., Jirjis, M.B., Schmit, B.D., Wang, M.C., Ulmer, J.L., and Kurpad, S.N. (2014). Diffusion tensor imaging of the spinal cord: insights from animal and human studies. Neurosurgery 74, 1-8.

Wilson, J.R., Forgione, N., and Fehlings, M.G. (2013). Emerging therapies for acute traumatic spinal cord injury. CMAJ 185, 485-492.

Xiao, Z.F., Chen, B., and Dai, J.W. (2016). Building the regenerative microenvironment with functional biomaterials for spinal cord injury repair. J Spine doi: 10.4172/2165-7939.S7-005.

Yiu, G., and He, Z. (2006). Glial inhibition of CNS axon regeneration. Nat Rev Neurosci 7, 617-627.

Yoon, S.H., Shim, Y.S., Park, Y.H., Chung, J.K., Nam, J.H., Kim, M.O., Park, H.C., Park, S.R., Min, B.H., Kim, E.Y., Choi, B.H., Park, H., and Ha, Y. (2007). Complete spinal cord injury treatment using autologous bone marrow cell transplantation and bone marrow stimulation with granulocyte macrophage-colony stimulating factor: Phase I/II clinical trial. Stem Cells 25, 2066-2073.

Yoshii, S., Oka, M., Shima, M., Taniguchi, A., Taki, Y., and Akagi, M. (2004). Restoration of function after spinal cord transection using a collagen bridge. J Biomed Mater Res A 70A, 569-575.

Zhao, R.R., and Fawcett, J.W. (2013). Combination treatment with chondroitinase $\mathrm{ABC}$ in spinal cord injury-breaking the barrier. Neurosci Bull 29, 477-483

Open Access This article is distributed under the terms of the Creative Commons Attribution License which permits any use, distribution, and reproduction in any medium, provided the original author(s) and source are credited. 